
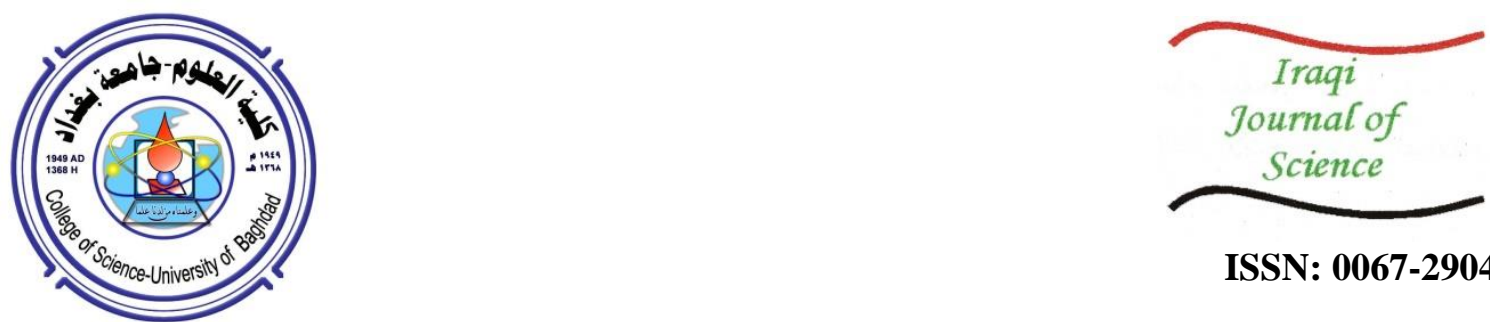

ISSN: 0067-2904

\title{
The Effects of Media Coverage on the Dynamics of Disease in Prey- Predator Model
}

\author{
Walaa Madhat Alwan*, Huda Abdul Satar \\ Department of Mathematics, College of Science, University of Baghdad, Baghdad, Iraq
}

Received: 3/6/2020

Accepted: $15 / 7 / 2020$

\begin{abstract}
In this paper, an eco-epidemiological model with media coverage effects is established and studied. An SIS-type of disease in predator is considered. All the properties of the solution of the proposed model are discussed. An application to the stability theory was carried out to investigate the local as well as global stability of the system. The persistence conditions of the model are determined. The occurrence of local bifurcation in the model is studied. Further investigation of the global dynamics of the model is achieved through using a numerical simulation.
\end{abstract}

Keywords: Media coverage, prey-predator, disease, stability, persistence, bifurcation.
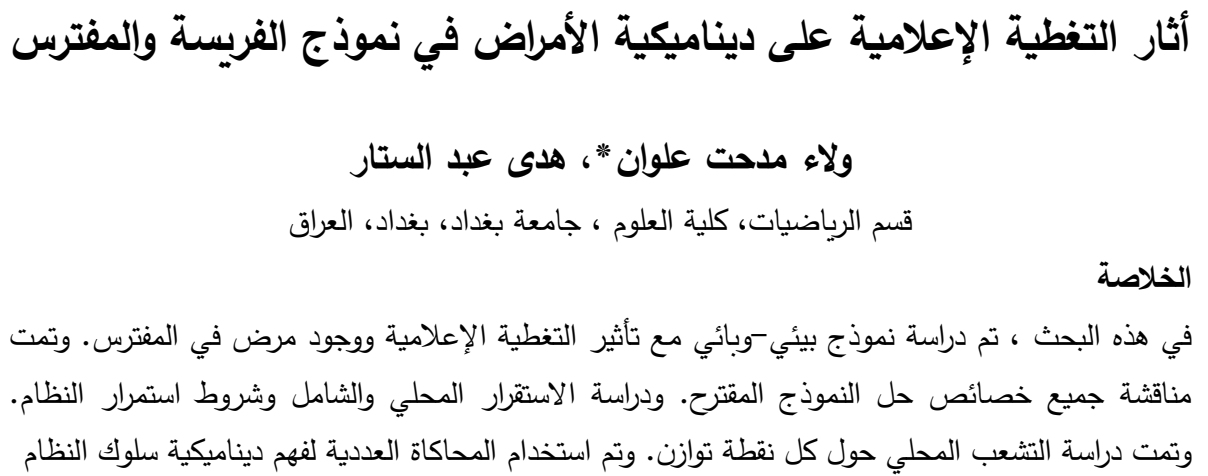

\section{Introduction}

The term eco-epidemiological models is used to describe the models that incorporate disease in ecological communities [1]. The first eco-epidemiological model including infectious diseases in the prey was introduced by Anderson and May [2]. Later on, a number of researchers proposed and studied eco-epidemiological models involving many biological factors [3-10]. It is observed that the spread of disease among the population is a main reason for the species extinction. Although many studies showed interest in eco-epidemiology. The impact of the media coverage, which has an important role in the outbreak of the infectious diseases, have been mostly neglected in the previous research papers.

The spreading and controlling of a susceptible-infected-recovered-susceptible (SIRS) disease with the media coverage was investigated by Liu and Cui [11]. Cui et al. [12] constructed a mathematical model that incorporates media coverage to understand its effects on the spread of infectious diseases in a given population. They concluded that more extensive media coverage in a given population leads to reduce the number of infected individuals. It is observed that the use of media coverage alert causes a reduction in the spread of AIDS, due to reducing the contact between human beings [13]. A similar

*Email: w.m.88ww@gmail.com 
observation was obtained regarding the spread of the severe acute respiratory syndrome (SARS) during 2002 and 2004 [14, 15]. Later on, some authors studied the effects of media coverage on the spread of infectious diseases [16]. Recently, Li et al. [17] proposed an epidemic model with media to describe the spread of infectious diseases in a given region. They found that media coverage plays an important role in controlling the spread of the disease. Al Basir [18] formulated and analyzed an epidemic model on the prevalence of infectious diseases using awareness campaign driven by media, with the aim of investigating the effects of awareness and delay on disease outbreak. These studies observed that effective media coverage can postpone the arrival of the infections peak and that a fewer number of individuals become infected in the course of transmission. Since the real-world system contains many species that interact with each other in different ways

, we intended in this paper to study the influence of media coverage on the dynamics of infectious diseases in prey-predator model. Consequently, a prey-predator model having disease in predator species and involving media coverage is proposed. Lotka-Volterra functional response is used for describing the predation process. Moreover, this paper is organized as follows. Section 2 deals with the model formulation. Section 3 determines the equilibrium points (EPs) and their local stability analysis. The global stability for the EPs is studied with the help of Lyapunov method (LM) in section 4. The bifurcation analysis of the system is investigated in section 5. Section 6 deals with the numerical simulation of the system. Finally, the discussion and conclusions are addressed in section 7 .

\section{The Mathematical model}

In this section, the effects of media coverage on an eco-epidemiological model dynamics are studied. The model consists of an infectious disease of SIS type in predator species that feeds on a prey. It is assumed that the prey is consumed by the predator according to Lotka-Voltera types of functional responses. Thus, in order to represent the dynamics of such a real-life system, the following hypotheses are adopted.

Let the variables $X(T), S(T)$ and $I(T)$ represent the densities at time $T$ for the prey, susceptible predator, and infected predator, respectively. It is assumed that $X(T)$ grows logistically with $r>0$ as the intrinsic growth rate and $K>0$ as the carrying capacity. The species $X(T)$ is consumed by the species $S(T)$ and $I(T)$ using Lotka-Volterra functional responses, with maximum attack rates of $a_{1}>0$ and $a_{2}>0$ and conversion rates of $e_{1} \in(0,1)$ and $e_{2} \in(0,1)$, respectively. The term $\left(b_{1}-b_{2} \frac{I}{m+I}\right)$ represents the infection rate [11] due to the direct contact between $S(T)$ and $I(T)$, where $b_{1}>0$ is the contact rate before media coverage alert, while $b_{2} \frac{I}{m+I}$ represents the reduced value in the contact due to media coverage alert, so that $b_{2}>0$ is the maximum transmission rate under the media coverage and $m>0$ is the non-response rate of individual's to the media coverage. Furthermore, since it is well known that the media coverage cannot prevent the spreading disease completely, then from now onward we take $b_{1} \geq b_{2}$. Also, the infected individuals may recover with a rate of $c>0$. Finally, the natural death rate of predator individuals is given by $d>0$, while the disease death rate is represented by $\alpha>0$.

According to the above hypotheses, the dynamics of the above-described system, that consists of a prey-predator system, which incorporates the media coverage, can be described in the following set of differential equations:

$$
\begin{aligned}
& \frac{d X}{d T}=r\left(1-\frac{X}{K}\right) X-a_{1} X S-a_{2} X I, \\
& \frac{d S}{d T}=e_{1} a_{1} X S+e_{2} a_{2} X I-\left(b_{1}-b_{2} \frac{I}{m+I}\right) S I+c I-d S, \\
& \frac{d I}{d T}=\left(b_{1}-b_{2} \frac{I}{m+I}\right) S I-(d+\alpha+c) I,
\end{aligned}
$$

with $X(T) \geq 0, S(T) \geq 0$ and $I(T) \geq 0$ as initial conditions. Therefore, system (1) has the domain $\mathbb{R}_{+}^{3}=\left\{(X, S, I) \in \mathbb{R}^{3} \mid X \geq 0, S \geq 0, I \geq 0\right\}$.

Clearly, system (1) contains $C^{1}$ functions and, therefore, these functions are Lipschitzain. Hence, the solution of system (1) exists and is unique. Furthermore, the uniform boundedness of the solutions of system (1) is proved in the following theorem.

Theorem (1). All the solutions of the system (1) are uniformly bounded.

Proof: We define $W_{1}=X+S+I$, then $\frac{d W_{1}}{d T}$ can be written as 
$\frac{d W_{1}}{d T} \leq 2 r\left(1-\frac{X}{2 K}\right) X-r X-d S-(d+\alpha) I \leq r K-\mu W_{1}$,

here, $\mu=\min \{r, d\}$, then direct computation shows that, for $T$ to go to $\infty$, we have $W_{1} \leq \frac{r K}{\mu}$. Therefore, all the variables are bounded.

\section{Existence of EPs and Their Local Stability Analysis}

It is observed that system (1) has four nonnegative biologically reasonable equilibrium points (EPs). The existence conditions for each of these EPs are established as the following:

The trivial EP, $P_{0}=(0,0,0)$, always exists.

The axial EP, $P_{1}=(K, 0,0)$, always exists as the prey population grows to carrying capacity in the absence of predation.

The infected predator free EP, $P_{2}=(\bar{X}, \bar{S}, 0)$, where

$$
\bar{X}=\frac{d}{e_{1} a_{1}} \text { and } \bar{S}=\frac{r\left(e_{1} a_{1} K-d\right)}{e_{1} a_{1}^{2} K},
$$

which exists under the condition

$$
e_{1} a_{1} K>d \text {. }
$$

The coexistence or positive EP, $P_{3}=\left(X^{*}, S^{*}, I^{*}\right)$, is given by

$$
S^{*}=\frac{A_{1}}{\left(b_{1}-b_{2} \frac{I^{*}}{m+I^{*}}\right)},
$$

where $A_{1}=d+\alpha+c$. While $\left(X^{*}, I^{*}\right)$ represents the positive intersection point of the following two isoclines

$$
\begin{aligned}
& g_{1}(X, I)=r\left(1-\frac{X}{K}\right)-a_{1} \frac{A_{1}}{\left(b_{1}-b_{2} \frac{I}{m+1}\right)}-a_{2} I=0, \\
& g_{2}(X, I)=e_{1} a_{1} X+e_{2} a_{2} \frac{\left(b_{1}-b_{2} \frac{I}{m+I}\right) X I}{A_{1}} \\
& -\left(b_{1}-b_{2} \frac{I}{m+I}\right) I+c \frac{\left(b_{1}-b_{2} \frac{I}{m+I}\right) I}{A_{1}}-d=0 .
\end{aligned}
$$

Obviously, as $I \rightarrow 0$, then the isoclines becomes

$$
\begin{aligned}
& g_{1}(X)=r\left(1-\frac{X}{K}\right)-a_{1} \frac{A_{1}}{b_{1}}=0, \\
& g_{2}(X)=e_{1} a_{1} X-d=0 .
\end{aligned}
$$

Therefore, $g_{1}(X)$ intersects the $X$-axis at the positive point $x_{1}=\frac{K\left(b_{1} r-a_{1} A_{1}\right)}{r b_{1}}$, however $g_{2}(X)$ intersects the $X$-axis at the positive point $x_{2}=\frac{d}{e_{1} a_{1}}$. Hence, the two isoclines (3b) and (3c) have a unique positive intersection point and then $P_{3}$ exists in the interior of $\mathbb{R}_{+}^{3}$ uniquely, if the next conditions hold.

$$
\begin{aligned}
& a_{1} A_{1}<b_{1} r, \\
& x_{1}<x_{2}, \\
& \frac{d I}{d X}=-\frac{\partial g_{1} / \partial X}{\partial g_{1} / \partial I}>0, \\
& \frac{d I}{d X}=-\frac{\partial g_{2} / \partial X}{\partial g_{2} / \partial I}<0 .
\end{aligned}
$$

Consequently, in the following, the stability near these EPs is studied locally using the linearization method. Note that it is easy to confirm that the Jacobian matrix (JM) of system (1) about an arbitrary point $(X, S, I)$ is

$J(X, S, I)=$

$$
\left[\begin{array}{ccc}
r-\frac{2 r X}{K}-a_{1} S-a_{2} I & -a_{1} X & -a_{2} X \\
e_{1} a_{1} S+e_{2} a_{2} I & e_{1} a_{1} X-\left(b_{1}-b_{2} \frac{I}{m+I}\right) I-d & e_{2} a_{2} X-b_{1} S+\frac{b_{2}(2 m+I) S I}{(m+I)^{2}}+c \\
0 & \left(b_{1}-b_{2} \frac{I}{m+I}\right) I & b_{1} S-\frac{b_{2}(2 m+I) S I}{(m+I)^{2}}-A_{1}
\end{array}\right] .(5)
$$

Now, it is obtained that the JM of system (1) around trivial EP, $P_{0}=(0,0,0)$, has the following eigenvalues:

$$
\lambda_{01}=r, \lambda_{02}=-d, \lambda_{03}=-A_{1} .
$$

Hence, the trivial EP is unstable (saddle point).

The JM of the system (1) at the axial EP, $P_{1}=(K, 0,0)$, can be written as: 


$$
J\left(P_{1}\right)=\left[\begin{array}{ccc}
-r & -a_{1} K & -a_{2} K \\
0 & e_{1} a_{1} K-d & e_{2} a_{2} K+c \\
0 & 0 & -A_{1}
\end{array}\right] .
$$

Therefore, the eigenvalues of $J\left(P_{1}\right)$ are given by

$$
\lambda_{11}=-r<0, \lambda_{12}=e_{1} a_{1} h-d, \lambda_{13}=-A_{1}<0 .
$$

Hence, the EP, $P_{1}=(K, 0,0)$, is locally asymptotically stable (LAS) if the condition below holds.

$$
e_{1} a_{1} K<d \text {. }
$$

Now, the JM of system (1) at the EP, $P_{2}=(\bar{X}, \bar{S}, 0)$, can be written as:

$$
J\left(P_{2}\right)=\left[\begin{array}{ccc}
-\frac{r d}{K e_{1} a_{1}} & -\frac{d}{e_{1}} & -\frac{a_{2} d}{e_{1} a_{1}} \\
\frac{r\left(e_{1} a_{1} K-d\right)}{a_{1} K} & 0 & e_{2} a_{2} \bar{X}-b_{1} \bar{S}+c \\
0 & 0 & b_{1} \bar{S}-A_{1}
\end{array}\right] .
$$

Clearly, one of the eigenvalue is $\lambda_{23}=b_{1} \bar{S}-A_{1}$ and the other two eigenvalues are the roots of the equation:

$$
\lambda_{2}^{2}-T \lambda_{2}+D=0
$$

where $T=-\frac{2 r d}{K e_{1} a_{1}}<0$ and $D=\frac{r d\left(K e_{1} a_{1}-d\right)}{K e_{1}^{2} a_{1}}>0$. Clearly, Eq. (9b) has the following roots

$$
\lambda_{21}=\frac{T}{2}+\frac{1}{2} \sqrt{T^{2}-4 D} ; \lambda_{22}=\frac{T}{2}-\frac{1}{2} \sqrt{T^{2}-4 D} .
$$

Hence, all these eigenvalues have negative real parts and hence $P_{2}$ is LAS if and only if the following condition holds:

$$
b_{1} \bar{S}<A_{1} \text {. }
$$

Finally, the JM evaluated at the positive EP, $P_{3}$, is given by

where:

$$
J\left(P_{3}\right)=\left(a_{i j}\right)_{3 \times 3},
$$

$$
\begin{aligned}
& a_{11}=r-\frac{2 r X^{*}}{K}-a_{1} S^{*}-a_{2} I^{*}, a_{12}=-a_{1} X^{*}, a_{13}=-a_{2} X^{*}, \\
& a_{21}=e_{1} a_{1} S^{*}+e_{2} a_{2} I^{*}, a_{22}=e_{1} a_{1} X^{*}-\left(b_{1}-\frac{b_{2} I^{*}}{m+I^{*}}\right) I^{*}-d, \\
& a_{23}=e_{2} a_{2} X^{*}-b_{1} S^{*}+\frac{b_{2}\left(2 m+I^{*}\right) S^{*} I^{*}}{\left(m+I^{*}\right)^{2}}+c, a_{31}=0, \\
a_{32}= & \left(b_{1}-\frac{b_{2} I^{*}}{m+I^{*}}\right) I^{*}, a_{33}=b_{1} S^{*}+\frac{b_{2}\left(2 m+I^{*}\right) S^{*} I^{*}}{\left(m+I^{*}\right)^{2}}-A_{1} .
\end{aligned}
$$

Then the characteristic equation of $J\left(P_{3}\right)$ can be written as:

where $C_{1}=-\left(a_{11}+a_{22}+a_{33}\right)$,

$$
\lambda_{3}^{3}+C_{1} \lambda_{3}^{2}+C_{2} \lambda_{3}+C_{3}=0
$$

with

$$
\begin{aligned}
& C_{2}=\left(a_{11} a_{22}-a_{12} a_{21}\right)+a_{11} a_{33}+\left(a_{22} a_{33}-a_{23} a_{32}\right), \\
& C_{3}=-\left[a_{11}\left(a_{22} a_{33}-a_{23} a_{32}\right)-a_{21}\left(a_{12} a_{33}-a_{13} a_{32}\right)\right],
\end{aligned}
$$

$$
\begin{gathered}
\Delta=C_{1} C_{2}-C_{3}=-\left(a_{11}+a_{22}\right)\left(a_{11} a_{22}-a_{12} a_{21}\right) \\
-a_{11} a_{33}\left(a_{11}+a_{33}\right)-\left(a_{22}+a_{33}\right)\left(a_{22} a_{33}-a_{23} a_{32}\right) \\
-2 a_{11} a_{22} a_{33}+a_{13} a_{21} a_{32} .
\end{gathered}
$$

Accordingly, by using the Routh-Hawirtiz criterion, all roots of Eq. (11b) have negative real part roots and, hence, the EP, $P_{3}$, is LAS if the following sufficient conditions are satisfied:

$$
\begin{aligned}
& r<\frac{2 r X^{*}}{K}+a_{1} S^{*}+a_{2} X^{*} I^{*}, \\
& e_{1} a_{1} X^{*}<\left(b_{1}-\frac{b_{2} I^{*}}{m+I^{*}}\right) I^{*}+d, \\
& e_{2} a_{2} X^{*}+\frac{b_{2}\left(2 m+I^{*}\right) S^{*} I^{*}}{\left(m+I^{*}\right)^{2}}+c<b_{1} S^{*}<A_{1}-\frac{b_{2} S^{*} I^{*}\left(2 m+I^{*}\right)}{\left(m+I^{*}\right)^{2}}, \\
& 2\left(r-\frac{2 r X^{*}}{K}-a_{1} S^{*}-a_{2} I^{*}\right)\left(e_{1} a_{1} X^{*}-\left(b_{1}-\frac{b_{2} I^{*}}{m+I^{*}}\right) I^{*}-d\right) \\
& \quad\left(b_{1} S^{*}+\frac{b_{2}\left(2 m+I^{*}\right) S^{*} I^{*}}{\left(m+I^{*}\right)^{2}}-A_{1}\right)<\left(-a_{2} X^{*}\right)\left(e_{1} a_{1} S^{*}+e_{2} a_{2} I^{*}\right)\left(\left(b_{1}-\frac{b_{2} I^{*}}{m+I^{*}}\right) I^{*}\right) .
\end{aligned}
$$

Now, we study the global stability and the persistence of system (1). It is well known that any biological system persists if and only if all its species persist for all the time. This means, from the 
mathematical point of view, that there is no trajectory that approaches asymptotically to the boundary axis or planes.

Now, according to system (1), if the infected individuals disappear, then the following subsystem is obtained

$$
\begin{aligned}
& \frac{d X}{d T}=r\left(1-\frac{X}{K}\right) X-a_{1} X S=h_{1}(X, S), \\
& \frac{d S}{d T}=e_{1} a_{1} X S-d S=h_{2}(X, S) .
\end{aligned}
$$

Clearly, subsystem (13) is a 2D system that has a unique positive point given by $(\bar{X}, \bar{S})$, which are given by Eq. (2a) and exist uniquely in the $X S$-plane under the condition (2b). According to the wellknown Poincare Bendixon theorem, the solution of system (13) approaches either to $\operatorname{EP}(\bar{X}, \bar{S})$ or else to the periodic dynamics. Now, by using the continuous function $g(X, S)=\frac{1}{X S}$, we obtain the following quantity

$$
\nabla=\frac{\partial\left(g h_{1}\right)}{\partial X}+\frac{\partial\left(g h_{2}\right)}{\partial S}=-\frac{r}{S K}<0 .
$$

Then, from the Dulac criterion, there is no periodic dynamics in the interior of the positive quadrant of the $X S$-plane. Therefore, according to the Poincare Bendixon theorem, the EP, $(\bar{X}, \bar{S})$, is a globally asymptotically stable (GAS) whenever it exists. Hence, the 3D system (1) has no periodic dynamics in the boundary planes.

Recall that the system (1) persists if and only if all the species coexist for all the future time. Hence, the following theorem contains the conditions that guarantee the persistence of the system.

Theorem (2). System (1) is uniformly persistent provided that:

$$
\begin{gathered}
e_{1} a_{1} K<d, \\
b_{1} \bar{S}>A_{1} .
\end{gathered}
$$

Proof: Suppose that $\pi$ is a point in the interior of $\mathbb{R}_{+}^{3}$ and $\gamma(\pi)$ is the orbit through $\pi$, and let $\Gamma(\pi)$ be the omega limit set of $\gamma(\pi)$. Furthermore, since $\Gamma(\pi)$ is bounded, due to the boundedness of the system (1), then we first show that $P_{0} \notin \Gamma(\pi)$.

Assume the contrary, since $P_{0}$ is a saddle point, then by Butler-McGhee lemma [19] there is at least one other point $u_{1}$ such that $u_{1} \in \omega^{s}\left(P_{0}\right) \cap \Gamma(\pi)$, where $\omega^{s}\left(P_{0}\right)$ is the stable manifold of $P_{0}$.

Now, since the stable manifold of $P_{0}$ is given by $S I$-plane and the entire orbit through $u_{1}$, say $\gamma\left(u_{1}\right)$, is contained in $\Gamma(\pi)$,

hence, if $u_{1}$ is on either boundary axes of $S I$-plane, then we obtain a contradiction to the boundedness of $\Gamma(\pi)$, due to the containment of the unbounded positive axis in it.

Now, let $u_{1}$ belongs to the interior of $S I$-plane. Since there is no EP in the interior of $S I$-plane, then the orbit through $u_{1}$, which is contained in $\Gamma(\pi)$, must be unbounded. This gives a contradiction and leads to $P_{0} \notin \Gamma(\pi)$.

Now, to proof that $P_{1} \notin \Gamma(\pi)$, we assume the converse. Since $P_{1}$ is a saddle point under the condition (14a), then by Butler-McGhee lemma, there is another point, say $u_{2}$, so that $u_{2} \in \omega^{s}\left(P_{1}\right) \cap \Gamma(\pi)$. Now, since the stable manifold of $P_{1}$ is given by $X I$-plane and the entire orbit through $u_{2}$ that denoted by $\gamma\left(u_{2}\right)$ is contained in $\Gamma(\pi)$ hence if $u_{2} \in \partial \mathbb{R}_{+(X I)}^{2}$ or $u_{2} \in \operatorname{Int} \mathbb{R}_{+(X I)}^{2}$, then a contradiction to the boundedness of $\Gamma(\pi)$ is obtained and then $P_{1} \notin \Gamma(\pi)$.

Finally, since the point $P_{2}$ is a saddle point under the condition (14b), then by using similar argument as given in the first part of the proof, we obtain that $P_{2} \notin \Gamma(\pi)$. Hence, the proof is complete.

\section{Global Stability Analysis}

In this section, the global stability of all the locally stable EPs is studied with the use of Lyapunov method, as shown in the following theorems.

Theorem (3). Assume that the EP, $P_{1}=(K, 0,0)$, of system (1) is LAS in $\mathbb{R}_{+}^{3}$, and the following conditions are satisfies:

Then it is GAS in $\mathbb{R}_{+}^{3}$.

$$
K<\min \left\{\frac{d}{a_{1}}, \frac{d+\alpha}{a_{2}}\right\} .
$$

Proof: Consider the following function:

$V_{1}=\left(X-K-K \ln \frac{X}{K}\right)+S+I$. 
Then $V_{1}$ is a $C^{1}$ function, which is a positive, definite, real valued function. Now, the function $\frac{d V_{1}}{d T}$ can be calculated as:

$$
\begin{gathered}
\frac{d V_{1}}{d T}=-\frac{r}{K}(X-K)^{2}-\left(1-e_{1}\right) a_{1} X S-\left(1-e_{2}\right) a_{2} X I \\
-\left(d-a_{1} K\right) S-\left(d+\alpha-a_{2} K\right) I .
\end{gathered}
$$

Since $e_{i} \in(0,1) ; i=1,2$, then we get

$$
\frac{d V_{1}}{d T}=-\frac{r}{K}(X-K)^{2}-\left(d-a_{1} K\right) S-\left(d+\alpha-a_{2} K\right) I .
$$

Obviously, under the condition (15), we have that $\frac{d V_{1}}{d T}$ is negative definite. Also, since $V_{1}$ is radially unbounded function, then $P_{1}$ is GAS.

Theorem (4). Assume that the EP, $P_{2}$, of system (1) is LAS in $\mathbb{R}_{+}^{3}$, and the following conditions are satisfied:

$$
\begin{aligned}
& e_{1} a_{2} \bar{X}+b_{1} \bar{S}<(d+\alpha), \\
& e_{2}<e_{1} .
\end{aligned}
$$

Then it is GAS in $\mathbb{R}_{+}^{3}$.

Proof: Consider the following function:

$$
V_{2}=\alpha_{1}\left(X-\bar{X}-\bar{X} \ln \frac{X}{\bar{X}}\right)+\alpha_{2}\left(S-\bar{S}-\bar{S} \ln \frac{S}{\bar{S}}\right)+\alpha_{3} I,
$$

where $\alpha_{i} ; i=1,2,3$ are positive constants that shall be determined later on. Clearly, $V_{1}: \mathbb{R}_{+}^{3} \rightarrow R$ is $C^{1}$ that is positive definite real valued function. Then we have

$$
\begin{aligned}
\frac{d V_{2}}{d T}= & -\frac{\alpha_{1} r}{K}(X-\bar{X})^{2}-a_{1}\left(\alpha_{1}-\alpha_{2} e_{1}\right)(X-\bar{X})(S-\bar{S}) \\
- & a_{2}\left(\alpha_{1}-\alpha_{2} e_{2}\right) X I-\left(\alpha_{2}-\alpha_{3}\right)\left(b_{1}-b_{2} \frac{I}{m+I}\right) S I-\frac{\alpha_{2}}{S} \bar{S} I \\
& \quad-\frac{\alpha_{2} e_{2} a_{2} \bar{S}}{S} X I-\left(\frac{\alpha_{2} d}{S}-\frac{\alpha_{2} e_{1} a_{1} \bar{X}}{S}\right)(S-\bar{S})^{2} \\
& -\left[\alpha_{3}(d+c+\alpha)-\alpha_{1} a_{2} \bar{X}-\alpha_{2} c-b_{1} \bar{S}\right] I-\frac{\alpha_{2} b_{2} I}{m+I} \bar{S} I .
\end{aligned}
$$

So, by choosing the positive constant as below:

$\alpha_{1}=e_{1}, \alpha_{2}=1$, and $\alpha_{3}=1$,

we obtain that:

$$
\begin{aligned}
\frac{d V_{2}}{d T}=- & \frac{r e_{1}}{K}(X-\bar{X})^{2}-a_{2}\left(e_{1}-e_{2}\right) X I-\frac{\bar{s}}{S} I-\frac{e_{2} a_{2} \bar{s}}{s} X I \\
- & {\left[(d+\alpha)-e_{1} a_{2} \bar{X}-b_{1} \bar{S}\right] I-\frac{b_{2} \bar{S}}{m+I} I^{2} . }
\end{aligned}
$$

Obviously, under the conditions (16a)-(16b), we have that $\frac{d V_{2}}{d T}$ is negative semi definite. Therefore, the $\mathrm{EP}, P_{2}$, is a stable point. Now, since $P_{2}$ is the only invariant set that satisfies $\frac{d V_{2}}{d T}=0$, then by using LaSalle's invariance principle, it is attracting. Hence, $P_{2}$ is GAS.

Theorem (5). Assume that the EP, $P_{3}=\left(X^{*}, Y^{*}, Z^{*}\right)$, of system (1) is LAS in $\mathbb{R}_{+}^{3}$, and the following conditions are satisfied:

$$
\left.\begin{array}{l}
\sigma_{12}^{2}<\sigma_{11} \sigma_{22} \\
\sigma_{13}^{2}<\sigma_{11} \sigma_{33} \\
\sigma_{23}^{2}<\sigma_{22} \sigma_{33}
\end{array}\right\},
$$

where all the symbol are defined in the proof. Then it is GAS in $\mathbb{R}_{+}^{3}$.

Proof: Consider the following function:

$V_{3}=d_{1}\left(X-X^{*}-X^{*} \ln \frac{X}{X^{*}}\right)+d_{2}\left(S-S^{*}-S^{*} \ln \frac{S}{S^{*}}\right)+d_{3}\left(I-I^{*}-I^{*} \ln \frac{I}{I^{*}}\right)$,

where $d_{i} ; i=1,2,3$ are positive constants that shall be determined later on. Clearly, $V_{1}: R_{+}^{3} \rightarrow R$ is a $C^{1}$ function that is a positive definite function. Then we have

$$
\begin{aligned}
\frac{d V_{3}}{d T}=- & \frac{d_{1} r}{k}\left(X-X^{*}\right)^{2}-\left[d_{1} a_{1}-d_{2} e_{1} a_{1}-\frac{d_{2} e_{2} a_{2} I^{*}}{S}\right]\left(X-X^{*}\right)\left(S-S^{*}\right) \\
- & {\left[d_{2} a_{2}\right]\left(X-X^{*}\right)\left(I-I^{*}\right)-\left[\frac{d_{2} a_{2} e_{2} X^{*} I^{*}}{S S^{*}}+\frac{d_{2} c I^{*}}{S S^{*}}\right]\left(S-S^{*}\right)^{2} } \\
& -\left[\left(d_{2}-d_{3} b_{1}-\frac{d_{2}\left(e_{2} a_{2} X+c\right)}{S}-\frac{d_{2} b_{2} m}{E E^{*}} I-\frac{\left(d_{2}-d_{3}\right) b_{2} I I^{*}}{E E^{*}}\right.\right. \\
& \left.-\frac{\left(d_{2}-d_{3}\right) b_{2} m I^{*}}{E E^{*}}\right]\left(S-S^{*}\right)\left(I-I^{*}\right)-\frac{d_{3} b_{2} m S}{E E^{*}}\left(I-I^{*}\right)^{2},
\end{aligned}
$$


where $E=m+I$ and $E^{*}=m+I^{*}$. So, by choosing $d_{1}=e_{1}, d_{2}=1$ and $d_{3}=1$, we get, after using the given condition (17), that:

$$
\begin{aligned}
\frac{d V_{3}}{d T} & \leq-\frac{1}{2}\left[\sqrt{\sigma_{11}}\left(X-X^{*}\right)+\sqrt{\sigma_{22}}\left(S-S^{*}\right)\right]^{2} \\
& -\frac{1}{2}\left[\sqrt{\sigma_{11}}\left(X-X^{*}\right)+\sqrt{\sigma_{33}}\left(I-I^{*}\right)\right]^{2} \\
& -\frac{1}{2}\left[\sqrt{\sigma_{22}}\left(S-S^{*}\right)-\sqrt{\sigma_{33}}\left(I-I^{*}\right)\right]^{2},
\end{aligned}
$$

here $\sigma_{11}=\frac{e_{1} r}{k}, \sigma_{22}=\frac{I^{*}}{S S^{*}}\left(e_{2} a_{2} X^{*}+c\right), \sigma_{33}=\frac{b_{2} m S}{E E^{*}}, \sigma_{12}=\frac{e_{2} a_{2} I^{*}}{S}, \sigma_{13}=a_{2}$ and $\sigma_{23}=\frac{\left(e_{2} a_{2} X+c\right)}{S}+$ $\frac{b_{2} m I}{E E^{*}}$.

Obviously, we have that $\frac{d V_{3}}{d T}$ is negative definite. Also, since $V_{3}$ is radially unbounded function, then $P_{3}$ is GAS.

\section{Local bifurcation}

It is well known that the bifurcation occurs if and only if there is a qualitative change in the behavior of the solution of a system, as occurs by varying the control parameter. Therefore in this section, a study of the occurrence of local bifurcation (LB) near the EPs of system (1) is performed using Sotomayor's theorem [20]. Also, it is well known that the non-hyperbolic property of an EP is a necessary but not sufficient condition for the occurrence of bifurcation in the neighborhood of that point. Therefore, the parameters, which change the EPs from hyperbolic to non-hyperbolic EPs, are considered as candidate bifurcation parameters of system (1), as shown in the next theorems. Now, for simplifying the notations, we rewrite system (1) in the vector form as follows

$\frac{d X}{d T}=F(X)$, with $X=(X, S, I)^{t}$ and $F=\left(F_{1}, F_{2}, F_{3}\right)^{t}$.

So, according to the JM of system (1) at the point $(X, S, I)$, it is easy to verify that for any vector $V=\left(v_{1}, v_{2}, v_{3}\right)^{t}$, we have that

where

$$
D^{2} \boldsymbol{F}(\boldsymbol{X})(V, V)=\left[\begin{array}{l}
\sum_{i, j=1}^{3} \frac{\partial F_{1}}{\partial x_{i} \partial x_{j}}\left(v_{i}\right)\left(v_{j}\right) \\
\sum_{i, j=1}^{3} \frac{\partial F_{2}}{\partial x_{i} \partial x_{j}}\left(v_{i}\right)\left(v_{j}\right) \\
\sum_{i, j=1}^{3} \frac{\partial F_{3}}{\partial x_{i} \partial x_{j}}\left(v_{i}\right)\left(v_{j}\right)
\end{array}\right]=\left[q_{i j}\right]_{3 \times 1},
$$

$q_{11}=-\frac{2 r}{K} v_{1}^{2}-2 a_{1} v_{1} v_{2}-2 a_{2} v_{1} v_{3}$,

$q_{21}=2 e_{1} a_{1} v_{1} v_{2}+2 e_{2} a_{2} v_{1} v_{3}-2\left[b_{1}-\frac{(2 m+I) b_{2} I}{(m+I)^{2}}\right] v_{2} v_{3}+\frac{2 b_{2} m^{2} S}{(m+I)^{3}} v_{3}^{2}$

$q_{31}=2\left[b_{1}-\frac{(2 m+I) b_{2} I}{(m+I)^{2}}\right] v_{2} v_{3}-\frac{2 b_{2} m^{2} s}{(m+I)^{3}} v_{3}^{2}$.

On other hand, we have also

where

$$
D^{3} \boldsymbol{F}(\boldsymbol{X})(V, V, V)=\left[n_{i j}\right]_{3 \times 1},
$$

$$
\begin{aligned}
& n_{11}=0, \\
& n_{21}=-\frac{6 b_{2} m^{2}}{(m+I)^{3}} v_{2} v_{3}^{2}+\frac{6 b_{2} m^{2}}{(m+I)^{4}} v_{3}{ }^{3}, \\
& n_{31}=-\frac{6 b_{2} m^{2}}{(m+I)^{3}} v_{2} v_{3}^{2}+\frac{6 b_{2} m^{2}}{(m+I)^{4}} v_{3}{ }^{3} .
\end{aligned}
$$

In the following theorems, the occurrence of LB around the EPs, $P_{1}, P_{2}$ and $P_{3}$, is investigated, respectively.

Theorem (6). Assume that the parameter $d$ satisfies that

$$
d \equiv d^{*}=e_{1} a_{1} K \text {. }
$$

Then system (1) near the EP, $P_{1}$, has a transcritical bifurcation (TB). However, saddle-node bifurcation (SNB) and pitchfork bifurcation (PB) cannot occur.

Proof: Note that, when $d=d^{*}$, then the JM of system (1) at $P_{1}$ can be written as

$$
J_{1}=J\left(P_{1}, d^{*}\right)=\left[\begin{array}{ccc}
-r & -a_{1} K & -a_{2} K \\
0 & 0 & e_{2} a_{2} K+c \\
0 & 0 & -A_{1}
\end{array}\right] \text {. }
$$


So, $J_{1}$ has the following eigenvalues: $\lambda_{11}^{*}=-r<0, \lambda_{12}^{*}=0$, and $\lambda_{13}^{*}=-A_{1}<0$ and, hence, the necessary but not sufficient condition for bifurcation is satisfied and $E_{1}$ is a nonhyperbolic point.

Let $\boldsymbol{V}_{\mathbf{1}}=\left(v_{11}, v_{12}, v_{13}\right)^{t}$ be the eigenvectors of $J_{1}$ corresponding to the eigenvalue $\lambda_{12}^{*}=0$. Then, simple computation gives that $\boldsymbol{V}_{\mathbf{1}}=\left(\gamma_{1} v_{12}, v_{12}, 0\right)^{T}$, where $v_{12}$ represents any nonzero real number and $\gamma_{1}=-\frac{a_{1} K}{r}<0$.

Also, let $\boldsymbol{\Psi}_{\mathbf{1}}=\left(\psi_{11}, \psi_{12}, \psi_{13}\right)^{T}$ represents the eigenvectors of $J_{1}{ }^{T}$ corresponding to the eigenvalue $\lambda_{12}^{*}=0$. Then again, simple calculation shows that $\boldsymbol{\Psi}_{\mathbf{1}}=\left(0, \psi_{12}, \gamma_{2} \psi_{12}\right)^{T}$, where $\psi_{13}$ is any nonzero real number and $\gamma_{2}=\frac{e_{2} a_{2} K+c}{A_{1}}>0$.

Since the partial derivative of vector field $\boldsymbol{F}$ with respect to the parameter $d$ is given by $\frac{\partial \boldsymbol{F}}{\partial d}=\boldsymbol{F}_{d}=$ $(0,-S,-I)^{T}$, hence by substituting $P_{1}$ and $d^{*}$ in this derivative, we obtain that $\boldsymbol{F}_{d}\left(P_{1}, d^{*}\right)=$ $(0,0,0)^{T}$.

Therefore $\boldsymbol{\Psi}_{\mathbf{1}}{ }^{t}\left[\boldsymbol{F}_{d}\left(E_{1}, d^{*}\right)\right]=0$.

Thus system (1) at $P_{1}$ with $d=d^{*}$ does not experience SNB in view of Sotomayor theorem. Moreover, since

$$
\boldsymbol{\Psi}_{\mathbf{1}}{ }^{t}\left[D \boldsymbol{F}_{d}\left(P_{1}, d^{*}\right) \boldsymbol{V}_{\mathbf{1}}\right]=-v_{12} \psi_{12} \neq 0,
$$

where $D \boldsymbol{F}_{d}$ represents the derivative of $\boldsymbol{F}_{d}$ with respect to $\boldsymbol{X}$, then $\boldsymbol{\Psi}_{\mathbf{1}}{ }^{t}\left[D^{2} \boldsymbol{F}\left(P_{1}, d^{*}\right)\left(\boldsymbol{V}_{\mathbf{1}}, \boldsymbol{V}_{\mathbf{1}}\right)\right]=2 e_{1} a_{1} \gamma_{1} \psi_{12} v_{12}{ }^{2} \neq 0$,

where $D^{2} \boldsymbol{F}$ represents the second derivative of $\boldsymbol{F}$ with respect to $\boldsymbol{X}$. Accordingly by Sotomayor theorem [20], system (1) near the EP, $P_{1}$, with $d=d^{*}$, possesses a TB, while PB cannot occur.

Theorem (7). Assume that

$$
\alpha \equiv \alpha^{*}=b_{1} \bar{S}-d-c .
$$

Then system (1) near the EP, $P_{2}$, has a TB, provided that the following condition holds

$$
b_{1} \beta_{2}-\frac{2 b_{2} \bar{S}}{m} \neq 0 \text {. }
$$

Otherwise it has a PB, provided that the following condition holds

$\beta_{2}-\frac{\bar{s}}{m} \neq 0$,

where all the symbols are given in proof.

Proof: Note that, when $\alpha=\alpha^{*}$, then the JM of system (1) at $P_{2}$ can be written as

$J_{2}=J\left(P_{2}, \alpha^{*}\right)=\left[\begin{array}{ccc}-\frac{r d}{K e_{1} a_{1}} & -\frac{d}{e_{1}} & -\frac{a_{2} d}{e_{1} a_{1}} \\ \frac{r\left(e_{1} a_{1} K-d\right)}{a_{1} K} & 0 & e_{2} a_{2} \bar{X}-b_{1} \bar{S}+c \\ 0 & 0 & 0\end{array}\right]=\left(d_{i j}\right)$.

Clearly, $J_{2}$ has zero eigenvalues, $\lambda_{23}{ }^{*}=0$, with the two other eigenvalues that are given by Eq. (9c) having negative real parts.

Let $\boldsymbol{V}_{\mathbf{2}}=\left(v_{21}, v_{22}, v_{23}\right)^{t}$ be the eigenvectors of $J_{2}$ corresponding to the eigenvalue $\lambda_{23}{ }^{*}=0$.

So, direct computation shows that $\boldsymbol{V}_{2}=\left(\beta_{1} v_{23}, \beta_{2} v_{23}, v_{23}\right)^{t}$, where $v_{23}$ represents any nonzero real number, $\beta_{1}=-\frac{d_{23}}{d_{21}}$, and $\beta_{2}=\frac{d_{11} d_{23}-d_{13} d_{21}}{d_{12} d_{21}}$.

Let $\boldsymbol{\Psi}_{2}=\left(\psi_{21}, \psi_{22}, \psi_{23}\right)^{t}$ represents the eigenvectors of $J_{2}{ }^{t}$ corresponding to the zero eigenvalue, $\lambda_{23}{ }^{*}=0$. Then straightforward calculation shows that $\boldsymbol{\Psi}_{2}=\left(0,0, \psi_{23}\right)^{t}$, where $\psi_{23}$ is any nonzero real number.

Since $\frac{\partial \boldsymbol{F}}{\partial \alpha}=\boldsymbol{F}_{\alpha}=(0,0,-I)^{t}$, hence we obtain that $\boldsymbol{F}_{\alpha}\left(P_{2}, \alpha^{*}\right)=(0,0,0)^{T}$.

Therefore, $\boldsymbol{\Psi}_{2}{ }^{t}\left[\boldsymbol{F}_{\alpha}\left(P_{2}, \alpha^{*}\right)\right]=0$.

Thus system (1) at the infected predator-free EP, $P_{2}$, with $\alpha=\alpha^{*}$ does not undergo SNB in view of Sotomayor theorem.

Now, since

$\boldsymbol{\Psi}_{2}^{t}\left[D \boldsymbol{F}_{\alpha}\left(P_{2}, \alpha^{*}\right) \boldsymbol{V}_{2}\right]=-v_{23} \psi_{23} \neq 0$,

and

$\boldsymbol{\Psi}_{2}{ }^{t}\left[D^{2} \boldsymbol{F}\left(P_{2}, \alpha^{*}\right)\left(\boldsymbol{V}_{2}, \boldsymbol{V}_{\mathbf{2}}\right)\right]=2 \psi_{23} v_{23}{ }^{2}\left[b_{1} \beta_{2}-\frac{b_{2} \bar{S}}{m}\right]$.

then, clearly, $\boldsymbol{\Psi}_{2}{ }^{t}\left[D^{2} \boldsymbol{F}\left(P_{2}, \alpha^{*}\right)\left(\boldsymbol{V}_{2}, \boldsymbol{V}_{2}\right)\right] \neq 0$, due to condition (22), and hence system (1) undergoes a TB near $P_{2}$ when $\alpha=\alpha^{*}$. However, violating condition (22) and using condition (23) leads to 
$\boldsymbol{\Psi}_{2}{ }^{t}\left[D^{3} \boldsymbol{F}\left(P_{2}, \alpha^{*}\right)\left(\boldsymbol{V}_{2}, \boldsymbol{V}_{2}, \boldsymbol{V}_{2}\right)\right]=-\frac{6 b_{2}}{m} \psi_{23} v_{23}{ }^{3}\left[\beta_{2}-\frac{\bar{s}}{m}\right] \neq 0$.

Hence system (1) undergoes a PB.

Theorem (8). Assume that conditions (12a)-(12b) along with the following conditions are satisfied

$$
\begin{aligned}
& b_{1} S^{*}<\min \left\{A_{1}-\frac{b_{2} S^{*} I^{*}\left(2 m+I^{*}\right)}{\left(m+I^{*}\right)^{2}}, e_{2} a_{2} X^{*}+\frac{b_{2}\left(2 m+I^{*}\right) S^{*} I^{*}}{\left(m+I^{*}\right)^{2}}+c\right\}, \\
& a_{22} a_{33}<a_{23} a_{32}<a_{11} a_{33}+a_{22} a_{33} .
\end{aligned}
$$

Then, when the parameter $r$ passes through the following value

$$
r^{*}=\frac{K}{\left(K-2 X^{*}\right)}\left[\frac{a_{21}\left(a_{12} a_{33}-a_{13} a_{32}\right)}{\left(a_{22} a_{33}-a_{23} a_{32}\right)}+a_{1} S^{*}+a_{2} I^{*}\right]
$$

system (1) near the coexistence EP, $P_{3}$, has a SNB, provided that the following condition holds

$$
\xi_{3} q_{11}{ }^{*}+\xi_{4} q_{21}{ }^{*}+q_{31}{ }^{*} \neq 0
$$

where all the symbols are given in the proof.

Proof: Straightforward computation shows that under the conditions (12a), (12b), (24a), (24b) and (24c) the coefficients of the characteristic equation given by Eq.(11b) are $C_{1}>0, C_{2}>0$ and $C_{3}=0$. Hence, Eq. (11b) has three roots (eigenvalues of $J\left(P_{3}\right)$ ) given by

$\lambda_{31}{ }^{*}=0, \lambda_{32}{ }^{*}=-\frac{C_{1}}{2}+\frac{1}{2} \sqrt{C_{1}{ }^{2}-4 C_{2}}, \lambda_{33}{ }^{*}=-\frac{C_{1}}{2}-\frac{1}{2} \sqrt{C_{1}{ }^{2}-4 C_{2}}$.

Clearly, the eigenvalues $\lambda_{32}{ }^{*}$ and $\lambda_{33}{ }^{*}$ have negative real parts. Hence the JM of system (1) around the point $P_{3}$ and $r=r^{*}$ can be written as $J_{3}=J\left(p_{3}, r^{*}\right)=\left(a_{i j}{ }^{*}\right)_{3 \times 3}$, with $a_{i j}{ }^{*}=a_{i j} ; \forall i, j$ and $a_{11}{ }^{*}=$ $a_{11}\left(r^{*}\right)$, where $a_{i j}$ are given in Eq. (11a). We will drop the star for simplification.

Let $\boldsymbol{V}_{\mathbf{3}}=\left(v_{31}, v_{32}, v_{33}\right)^{t}$ be the eigenvectors of $J_{3}$ corresponding to $\lambda_{31}{ }^{*}=0$. Then, direct computation shows that $\boldsymbol{V}_{3}=\left(\xi_{1} v_{33}, \xi_{2} v_{33}, v_{33}\right)^{t}$, where $v_{33}$ represents any nonzero real number, $\xi_{1}=\frac{a_{12} a_{23}-a_{13} a_{22}}{a_{11} a_{22}-a_{12} a_{21}}<0$, and $\xi_{2}=\frac{a_{21} a_{13}-a_{23} a_{11}}{a_{11} a_{22}-a_{12} a_{21}}$.

Let $\mathbf{\Psi}_{3}=\left(\psi_{31}, \psi_{32}, \psi_{33}\right)^{t}$ represents the eigenvectors of $J_{3}{ }^{t}$ corresponding to the eigenvalue $\lambda_{31}{ }^{*}=0$. Then simple calculation shows that $\boldsymbol{\Psi}_{3}=\left(\xi_{3} \psi_{33}, \xi_{4} \psi_{33}, \psi_{33}\right)^{t}$, where $\psi_{33}$ is any nonzero real number, $\xi_{3}=\frac{-a_{32} a_{11}}{a_{11} a_{22}-a_{12} a_{21}}>0$, and $\xi_{4}=\frac{a_{32} a_{21}}{a_{11} a_{22}-a_{12} a_{21}}>0$.

We have that $\frac{\partial \boldsymbol{F}}{\partial r}=\boldsymbol{F}_{r}=\left(X-\frac{X^{2}}{K}, 0,0\right)^{t}$, hence we obtain that $\boldsymbol{F}_{r}\left(P_{3}, r^{*}\right)=\left(X^{*}-\frac{X^{* 2}}{K}, 0,0\right)^{t}$. Therefore, we obtain that

$\boldsymbol{\Psi}_{3}{ }^{t}\left[\boldsymbol{F}_{r}\left(P_{3}, r^{*}\right)\right]=\xi_{3} \psi_{33}\left(X^{*}-\frac{X^{* 2}}{K}\right) \neq 0$.

Consequently, the first condition of SNB in view of Sotomayor theorem is satisfied. Now, since

$\boldsymbol{\Psi}_{3}{ }^{t}\left[D^{2} \boldsymbol{F}\left(P_{3}, r^{*}\right)\left(\boldsymbol{V}_{3}, \boldsymbol{V}_{3}\right)\right]=-2 v_{33}{ }^{2} \psi_{33}\left[\xi_{3} q_{11}{ }^{*}+\xi_{4} q_{21}{ }^{*}+q_{31}{ }^{*}\right]$,

where

$q_{11}{ }^{*}=\left[\frac{r^{*}}{K} \xi_{1}^{2}+a_{1} \xi_{1} \xi_{2}+a_{2} \xi_{1}\right]$,

$q_{21}^{*}=\left[-e_{1} a_{1} \xi_{1} \xi_{2}-e_{2} a_{2} \xi_{1}+\left(b_{1}-\frac{\left(2 m+I^{*}\right) b_{2} I^{*}}{\left(m+I^{*}\right)^{2}}\right) \xi_{2}-\frac{b_{2} m^{2} S^{*}}{\left(m+I^{*}\right)^{3}}\right]$,

$q_{31}{ }^{*}=\left[\frac{b_{2} m^{2} S^{*}}{\left(m+I^{*}\right)^{3}}-\left(b_{1}-\frac{\left(2 m+I^{*}\right) b_{2} I^{*}}{\left(m+I^{*}\right)^{2}}\right) \xi_{2}\right]$,

then, clearly, $\boldsymbol{\Psi}_{3}{ }^{T}\left[D^{2} \boldsymbol{F}\left(P_{3}, r^{*}\right)\left(\boldsymbol{V}_{3}, \boldsymbol{V}_{3}\right)\right] \neq 0$ under the condition (25), and hence system (1) undergoes a SNB near the coexistence equilibrium.

\section{Numerical Simulation}

In this section, the global dynamics of system (1) is further investigated. To specify the control set of parameters, the system is solved numerically. System (1) is solved numerically using Runge-Kutta of order six, followed by a four-step Predictor-Corrector method. Then, all the obtained numerical results are drawn in the form of $3 D$ phase portrait and $2 D$ time series using MATLAB version 6. Therefore, in order to run simulations, the following hypothetical set of biological data is used in this section.

$$
\begin{aligned}
r & =2, K=20, \quad a_{1}=1, a_{2}=0.75, e_{1}=0.7, \quad e_{2}=0.6, \\
b_{1} & =0.15, b_{2}=0.1, m=20, c=0.05, d=0.05, \alpha=0.01 .
\end{aligned}
$$

It is observed, for this set of data, that the system (1) approaches asymptotically to the unique coexistence EP, $E_{3}=(0.10,0.77,1.62)$, starting from three different initial values, as shown in the following two figures (Figures- 1 and 2). 


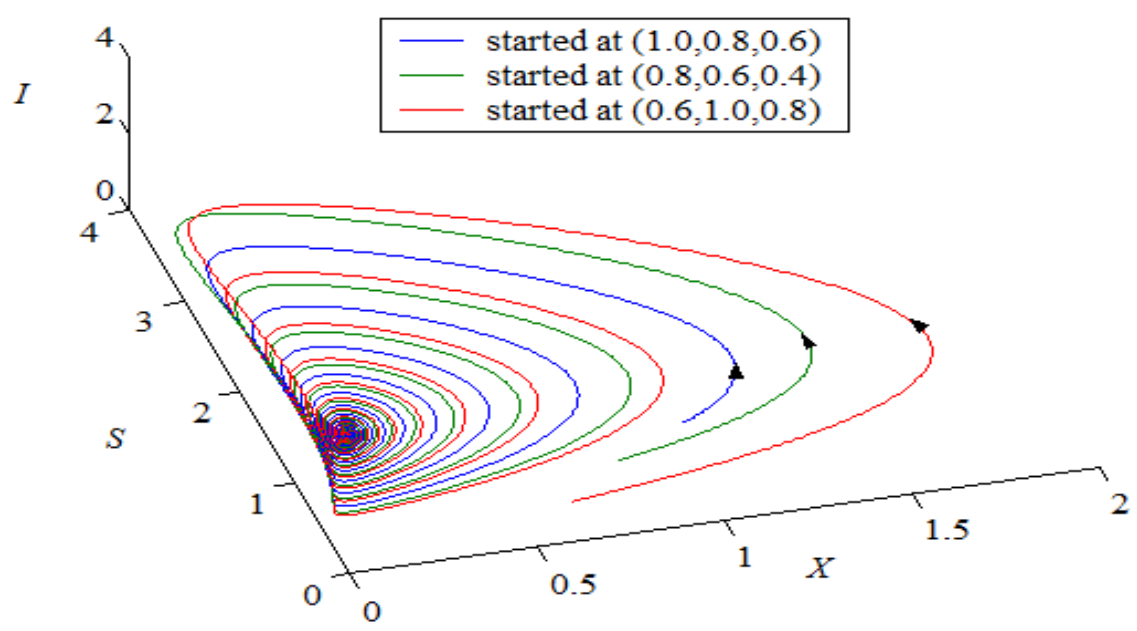

Figure 1-3D phase portrait of system (1) using the parameters given by Eq. (26) in which the solution approaches asymptotically to the $E_{3}=(0.10,0.77,1.62)$.
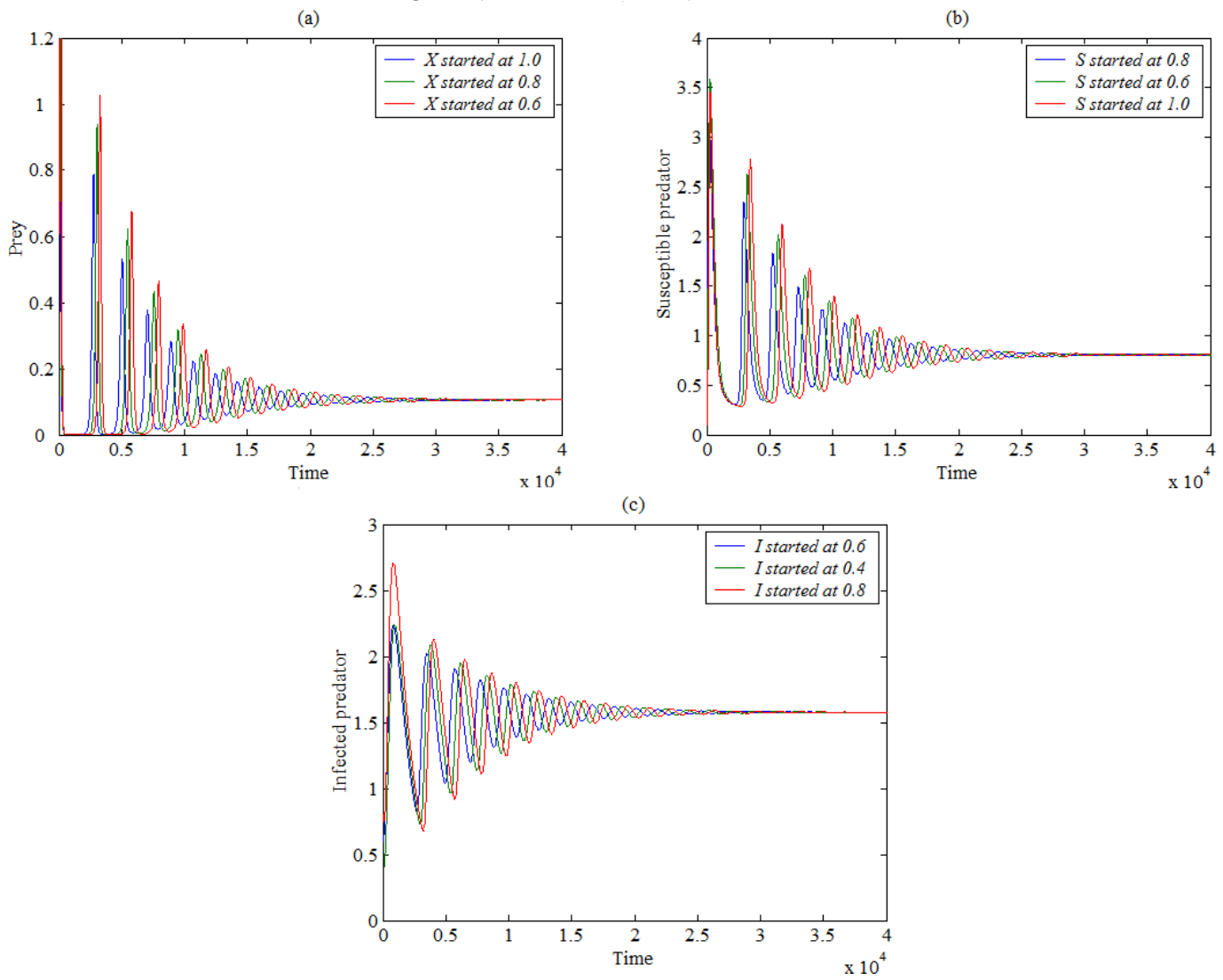

Figure 2-The solution of system (1) approaches asymptotically to $E_{3}=(0.10,0.77,1.62)$ for the data (26). (a) The trajectory of prey versus time. (b) The trajectory of susceptible predator versus time. (c) The trajectory of infected predator versus time.

According to these two figures, system (1) persists at the coexistence point in $\mathbb{R}_{+}^{3}$. Now, in order to discuss the effect of varying the parameters' values of system (1) on the dynamical behavior of the system, the system is solved numerically for the data given in Eq. (26) and then the obtained solutions are drawn as shown below. It is observed that, for the values of parameter $r$ in the range $r \leq 0.7$ with the other parameters as in Eq. (26), system (1) approaches asymptotically to infected predator-free EP in the interior of $X S$-plane, otherwise it has a GAS coexistence EP, see Figure-3a and Figure-3b for 
typical values of $r$. Now, the effect of varying the carrying capacity of the prey species is investigated numerically as shown in Figure-4).
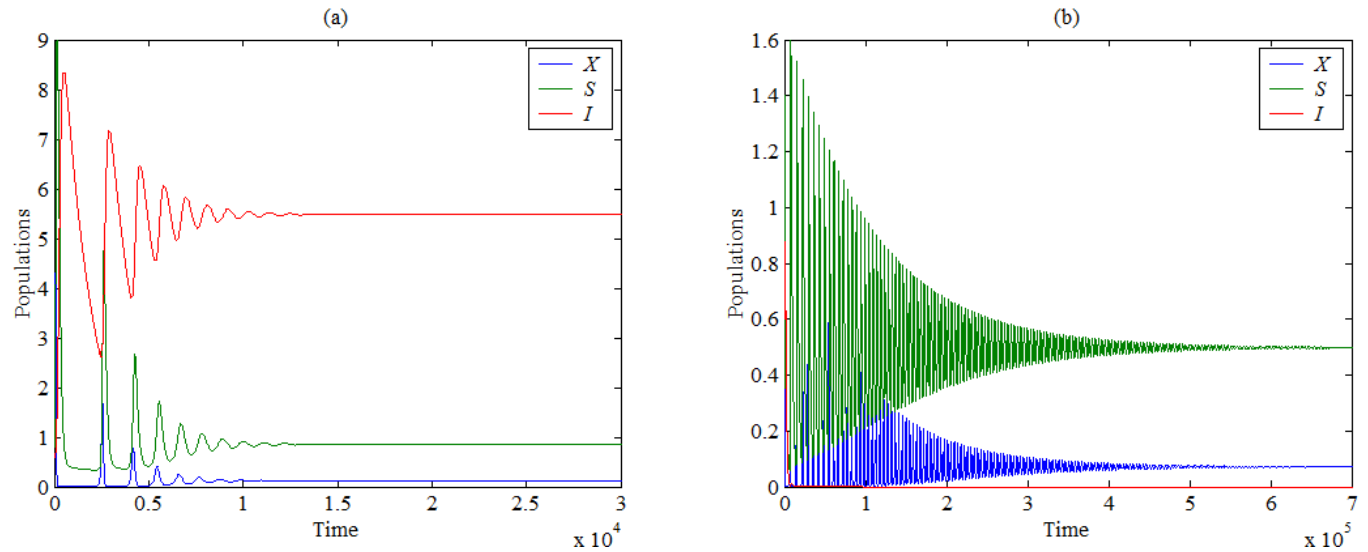

Figure 3-The trajectories of system (1) versus time for the data given by Eq. (26) with different values of $r$. (a) The system approaches asymptoticaly to $E_{3}=(0.12,0.85,5.48)$ when $r=5$. (b) The system approaches asymptotically to $E_{2}=(0.07,0.49,0)$ when $r=0.5$.
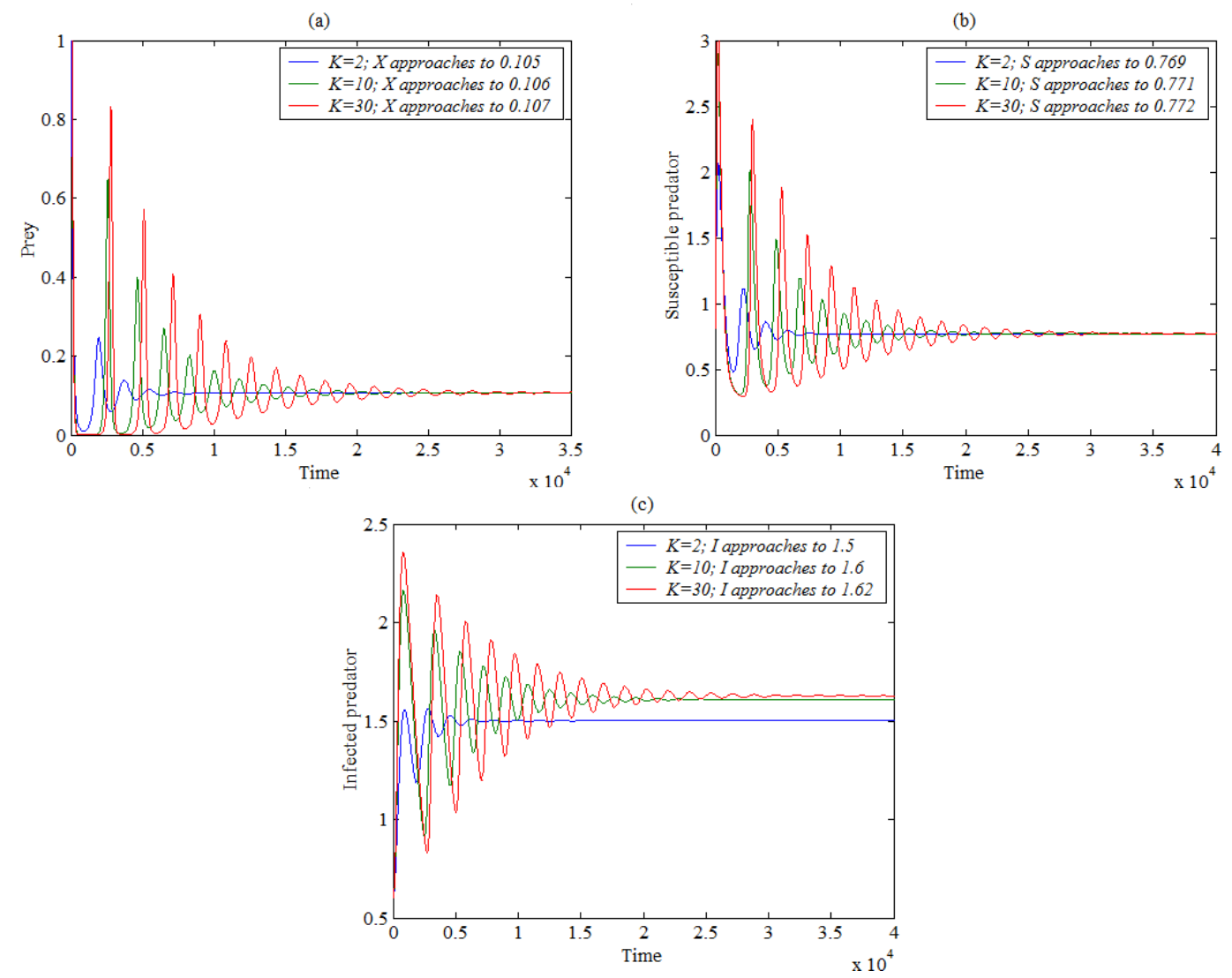

Figure 4-The trajectories of system (1) versus time for the data given by Eq. (26) with different values of K. (a) Trajectories of the prey. (b) Trajectories of the susceptible predator. (c) Trajectories of the infected predator.

Obviously, as the carrying capacity increases, the population, especially the infected predator, increases too, but the system still persists at the coexistence EP.

Now, varying $a_{1}$ in the range of $a_{1} \geq 2.8$ leads to an extinction in the infected predator and the system (1) approaches to $E_{2}$, as shown in the typical illustration given by Figure-5. While for $a_{1} \leq$ 0.7 , the system approaches to periodic dynamics in $3 \mathrm{D}$, as shown in the typical illustration given by Figure-6. Otherwise, system (1) still has a global coexistence EP. 
(a)

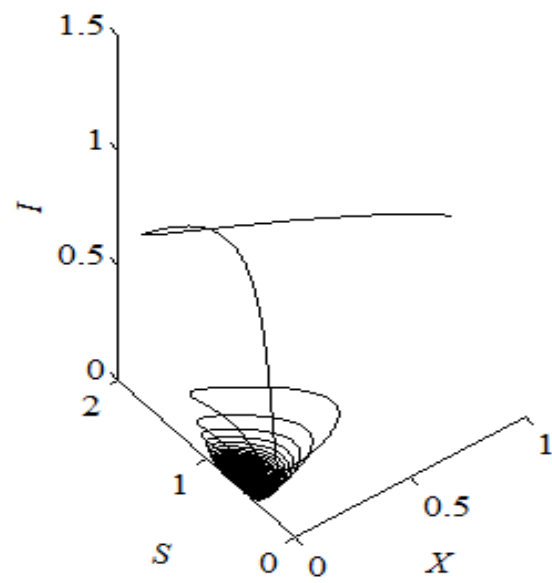

(b)

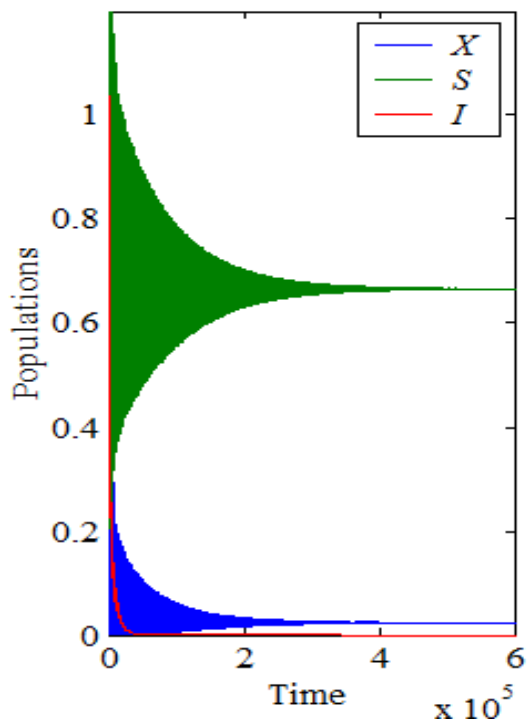

Figure 5-The solution of system (1) of system (1) for $\mathrm{a}_{1}=3$ with the other parameters as in Eq. (26), which approaches asymptoticaly to $E_{2}=(0.02,0.66,0)$. (a) $3 \mathrm{D}$ phase plot of the attractor. (b) The trajectories of each population versus time.

(a)

(b)

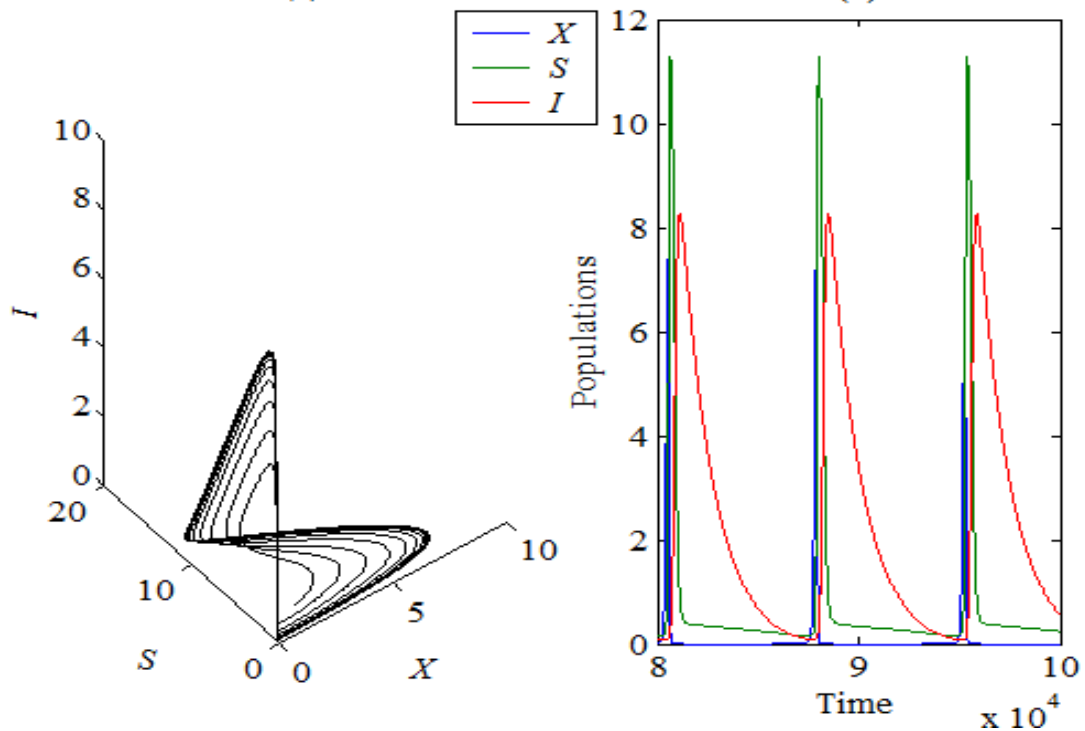

Figure 6-The solution of system (1) of system (1) for $\mathrm{a}_{1}=0.5$ with the other parameters as in Eq. (26), which approaches periodic in the interior of $\mathbb{R}_{+}^{3}$. (a) $3 \mathrm{D}$ phase plot of the attractor. (b) The trajectories of each population versus time.

Moreover, for the parameter $a_{2}$ in the range $a_{2} \geq 1.1$, it is observed that system (1) approaches to periodic dynamics in $3 \mathrm{D}$ as shown in the typical illustration given by Figure-7. However, it approaches to $E_{3}$ otherwise. 
(a)

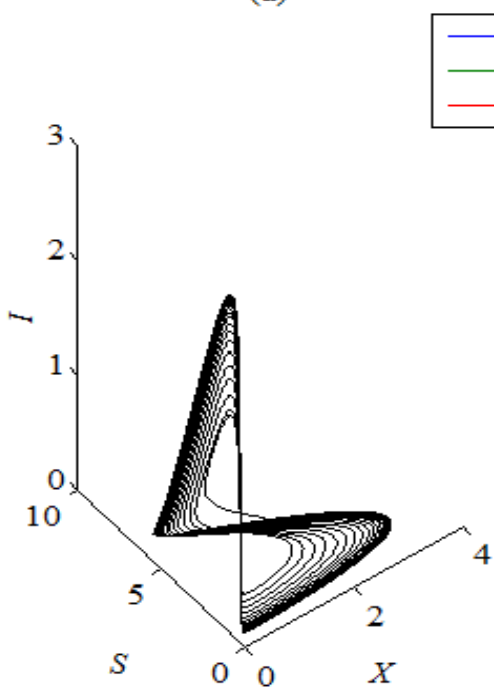

(b)

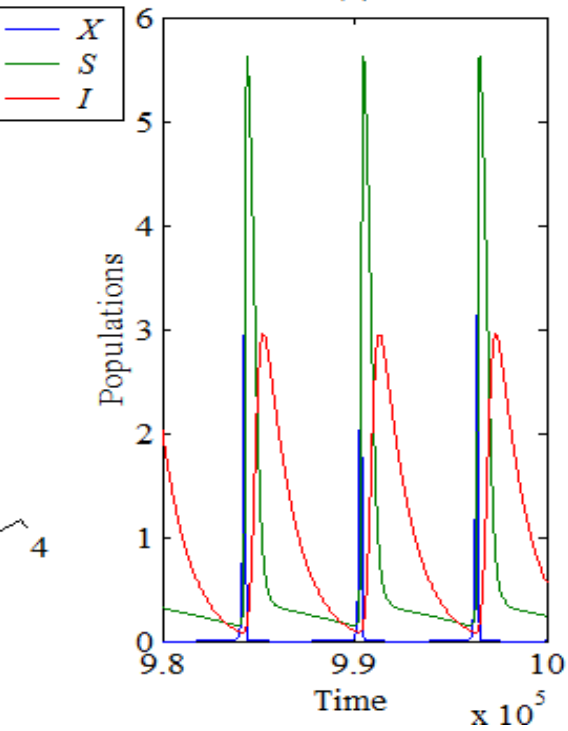

Figure 7-The solution of system (1) of system (1) for $\mathrm{a}_{2}=1.2$ with the other parameters as in Eq. (26), which approaches periodic in the interior of $\mathbb{R}_{+}^{3}$. (a) $3 \mathrm{D}$ phase plot of the attractor. (b) The trajectories of each population versus time.

It is observed that varying the parameters $e_{1}$ and $\alpha$ have similar effect as that shown with varying $a_{2}$. Now, for the parameter $e_{2}$ in the range $e_{2} \leq 0.1$, it is observed that system (1) approaches to periodic dynamics in $3 \mathrm{D}$, as shown in the typical illustration given by Figure-8. However, it approaches to $E_{3}$ otherwise.

(a)

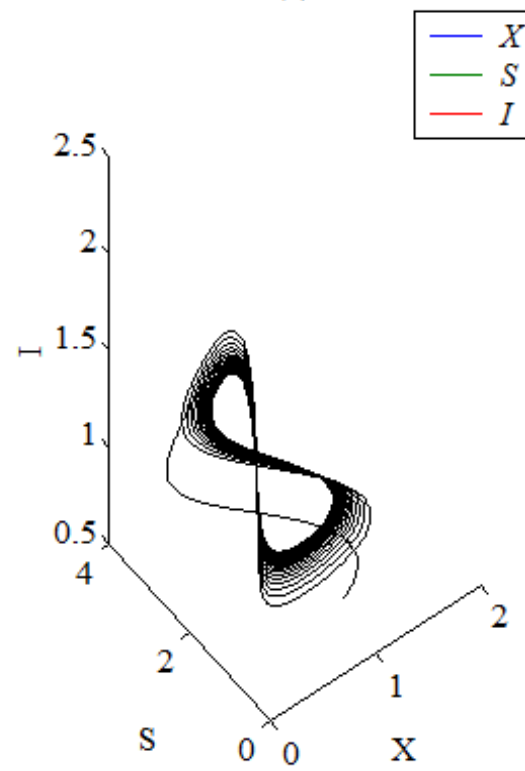

(b)

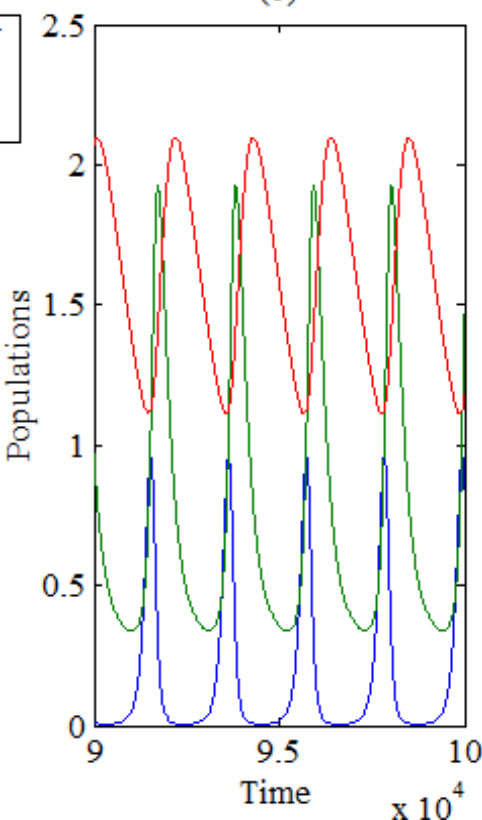

Figure 8-The solution of system (1) of system (1) for $\mathrm{e}_{2}=0.05$ with the other parameters as in Eq. (26), which approaches periodic in the interior of $\mathbb{R}_{+}^{3}$. (a) $3 \mathrm{D}$ phase plot of the attractor. (b) The trajectories of each population versus time.

On the other hand, varying the parameters of the infection rate of system (1) was also studied. It is observed that, for $b_{1} \geq 0.1$, system (1) has a GAS at $E_{3}$ with quantitative changes in the sizes of populations. However, for $b_{2}=0.15$ (maximum transmission rate under the media coverage alert), with increasing the response of individuals to the media coverage alert or decreasing the parameter $m$, 
system (1) approaches gradually to $E_{2}$, as shown in Figure-9 for the values $m=20,10,5,0$, respectively.

Finally, for the parameter $c$ in the range $c \geq 0.3$, with the rest of parameters being as in Eq. (26), system (1) approaches asymptoticaly to $E_{2}$, as shown in the typical illustration given by Figure-10. Otherwise system (1) still approaches to $E_{3}$ in the interior of $\mathbb{R}_{+}^{3}$. Similar effect was obtained, as that happened with varying $c$, when we varied the parameter $d$.

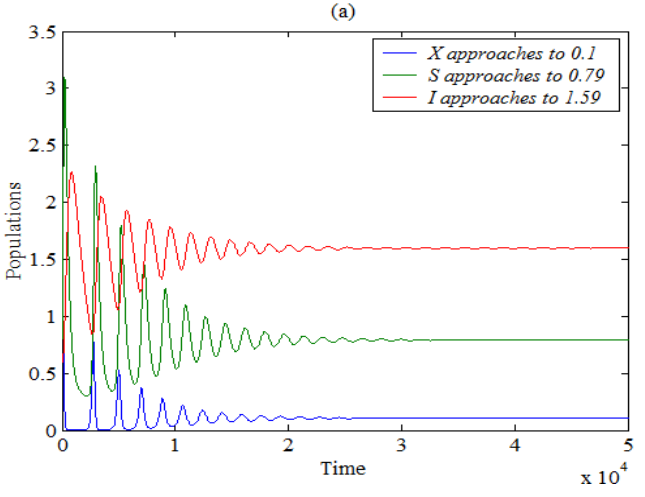

(c)

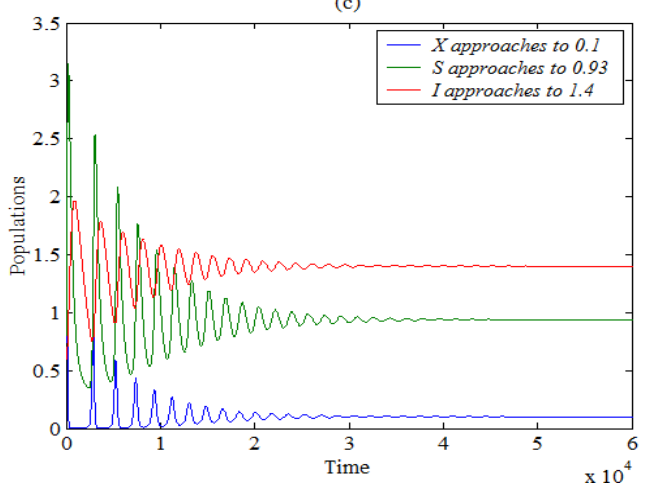

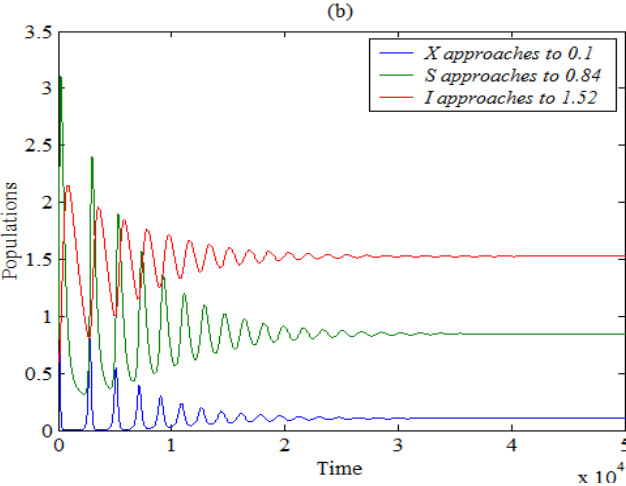

(d)

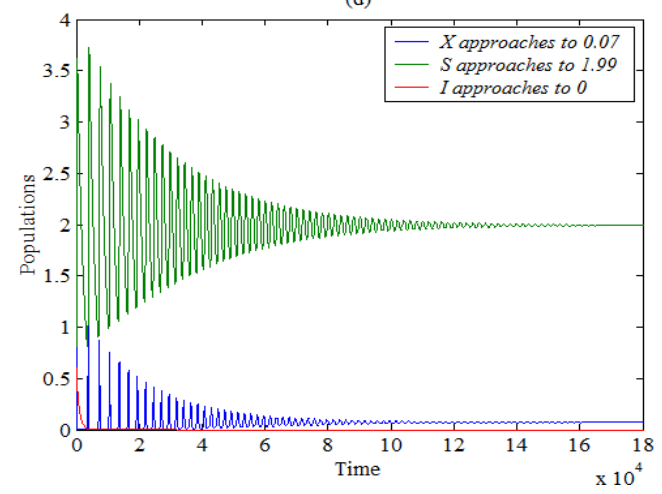

Figure 9-The trajectories of system (1) versus time for the data given by Eq. (26) with $\mathrm{b}_{2}=0.15$ and different values of $\mathrm{m}$. (a) The system approaches asymptotically to $E_{3}=(0.1,0.79,1.59)$ when $\mathrm{m}=20$. (b) The system approaches asymptotically to $E_{3}=(0.1,0.84,1.52)$ when $m=10$. (c) The system approaches asymptotically to $E_{3}=(0.1,0.93,1.4)$ when $m=5$. (d) The system approaches asymptotically to $E_{2}=(0.07,1.99,0)$ when $m=0$.

(a)

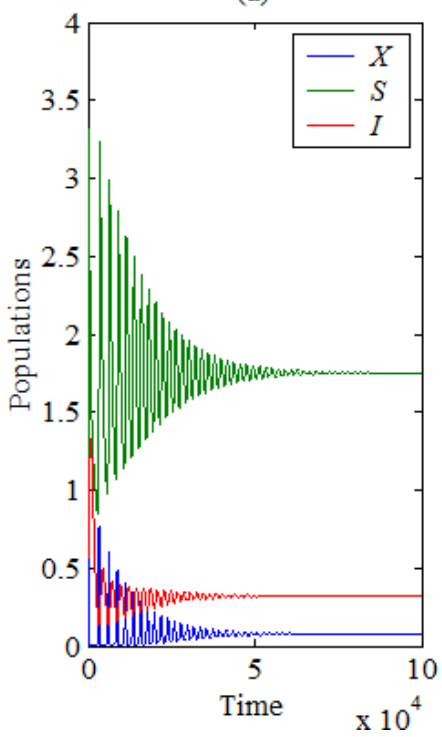

(b)

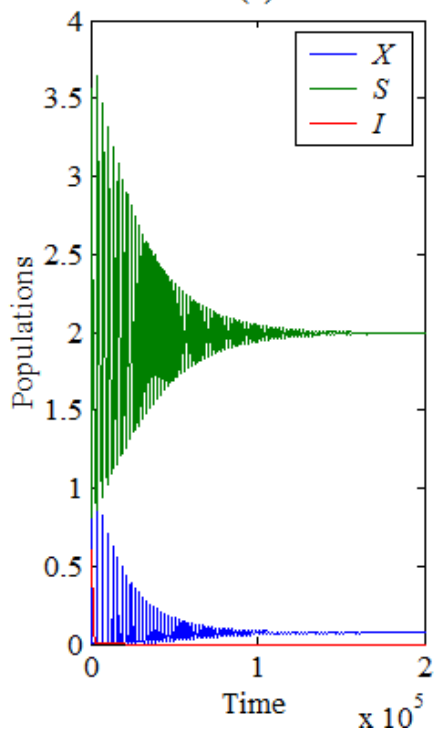

Figure 10-The trajectories of system (1) versus time for the data given by Eq. (26) with different values of $c$. (a) The system approaches asymptotically to $E_{3}=(0.07,1.75,0.32)$ when $c=0.2$. (b) The system approaches asymptotically to $E_{2}=(0.07,1.99,0)$ when $c=0.4$. 


\section{Discussion and conclusions}

In this paper, the impact of media coverage alert on the dynamical behavior of the diseased preypredator population and the spread of the disease were investigated. It is assumed that the system is consisting of prey that grows logistically and consumed by a predator according to Lotka-Voltter functional response. There is an infectious disease of SIS-type in the predator. The system of differential equations that describes the dynamics of the above system is constructed. All the solution properties are discussed. The stability analysis of the system is studied locally as well as globally. The conditions of system persistence are established. The occurrence of local bifurcation is also investigated. Finally, numerical simulation is carried out to further understand the global dynamics of the system using hypothetical set of parameters values. The following conclusions are obtained from the numerical simulations.

- System (1) has only two types of attractors that approach to EP or periodic dynamics.

- Decreasing the intrinsic growth rate below a specific value causes extinction in infected predator and the solution approaches to the infected predator-free EP. Otherwise it persists at a coexistence EP.

- Varying the carrying capacity or infection rate, due to contact between the susceptible individuals and infected individuals, before applying media coverage has quantitative effects on the size of population and the system still persist at the coexistence EP.

- Increasing the attack rate of prey by susceptible predator above a specific value leads to extinction in the infected predator and the solution approaches to infected predator-free EP. While by decreasing it below a specific value, the system loses its stability and still persists in the form of periodic attractor in the interior of $\mathbb{R}_{+}^{3}$. The solution still approaches to the coexistence EP otherwise.

- Increasing the attack rate of prey by infected predator above a specific value causes instability in the system and the solution approaches asymptotically to periodic attractor in the interior of $\mathbb{R}_{+}^{3}$. Again, the solution still approaches to coexistence EP otherwise. The conversion rate of the prey due to the attack by susceptible predator and the disease-associated death rate have similar effects as that obtained by the attack rate.

- Decreasing the conversion rate of the prey due to the attack by infected predator below a specific value causes instability in the system and the solution approaches asymptotically to periodic attractor in the interior of $\mathbb{R}_{+}^{3}$. The solution still approaches to coexistence EP otherwise

- Increasing the maximum transmission rate of disease under the media coverage alert to up to the maximum value $\left(b_{2} \equiv b_{1}\right)$ without increasing the response of individuals to that alert (or decreasing the non-response rate of individuals to the media coverage) do not stop the spread of disease. However, decreasing the rate of non-response of individuals up to zero causes stopping in the spread of disease and the system approaches to infected predator-free EP.

- Increasing the disease death rate above a specific value leads to losing the persistence of the system and the solution approaches asymptotically to the infected predator-free EP. Otherwise it still persists at a coexistence EP. A similar observation was obtained for the parameter that represents the natural death rate of the predator.

\section{References}

1. Venturino E. 2016. Eco-epidemiology: a more comprehensive view of population interactions. Mathematical Modelling of Natural Phenomena, 11(1): 49-90.

2. Anderson R.M. and May R.M. 1980. Infectious diseases and population cycles of forest insects. Science, 210(4470): 658-661.

3. Beretta E. and Kuang Y. 1998. Modeling and analysis of a marine bacteriophage infection, Mathematical Biosciences, 149(1): 57-76.

4. Chattopadhyay J. and Arino O. 1999. A predator-prey model with disease in the prey. Nonlinear Analysis, 36 (6): 747-766.

5. Bairagi N., Roy P.K., and Chattopadhyay J. 2007. Role of infection on the stability of a predatorprey system with several response functions - a comparative study, Journal of Theoretical Biology, 248(1): 10-25.

6. Bhattacharyya R. and Mukhopadhyay, B. 2010. On an eco-epidemiological model with prey harvesting and predator switching: local and global perspectives. Nonlinear Analysis Real World Applications, 11(455): 3824-3833. 
7. Bob, W., George, A.K. and Krishna, D. 2011. Stabilization and complex dynamics in a predatorprey model with predator suffering from an infectious disease. Ecological Complexity 8: 113-122 .

8. Liu, X. 2011. Bifurcation of an eco-epidemiological model with a nonlinear incidence rate. Applied Mathematics and Computation, 218: 2300-2309.

9. Naji R.K. \& Mustafa A.N. 2012. The Dynamics of an Eco-Epidemiological Model with Nonlinear Incidence Rate. Journal of Applied Mathematics 2012, Article ID 852631, pp. (24 pages).

10. Biswas, S., Samanta, S. and Chattopadhyaya, J. 2015. A model based theoretical study on cannibalistic prey-predator system with disease in both populations. Differential Equations and Dynamical Systems, 23: 327-370.

11. Liu Y. and Cui J. 2008. The impact of media coverage on the dynamics of infectious disease. International Journal of Biomathematics, 1(1): 65-74.

12. Cui J., Tao X. and Zhu H. 2008. An SIS infectious model incorporating media coverage. Rocky Mountain, 38(5): 1323-1334.

13. Rahman M.S. and Rahman L. 2007. Media and education play a tremendous in mounting ADIS awareness among married couples in Bangladesh. AIDS Research and Therapy, 4: 10-17.

14. Wang W. \& Ruan S. 2004. Simulating the SARS outbreak in Beijing with limited data. Journal of Theoretical Biology, 227: 369-379.

15. ZhouY., Ma Z. and Brauer F. 2004. A discrete epidemic model for SARS transmission and control in China. Mathematical and Computer Modelling, 40: 1491-1506.

16. Liu R., Wu J. and Zhu H. 2007. Media / psychological impact on multiple outbreaks of emerging infectious disease Computational and Mathematical Methods in Medicine, 8(3): 153-164.

17. Liu, M., Chang, Y. and Zuo, Y. 2016. Modelling the Impact of Media in Controlling the Diseases with a Piecewise Transmission Rate. Discrete Dynamics in Nature and Society 2016, Article ID 3458965, (6 pages).

18. Al Basir, F. 2018. Dynamics of Infectious Diseases with Media Coverage and Two Time Delay. Mathematical Models and Computer Simulations, 10(6): 770-783

19. Freedman H. and Waltoman, P. 1984. Persistence in models of three interacting predator prey populations. Mathematical Bioscience, 68: 213-231.

20. Perko L. 2013. Differential equations and dynamics systems. Third Edition, Springer-Verlag, New York. Inc. 